\title{
Antenna Selection for Full-Duplex MIMO Two-Way Communication Systems
}

\author{
Daniel G. Wilson-Nunn, Anas Chaaban, Member, IEEE, Aydin Sezgin, Senior Member, IEEE, and \\ Mohamed-Slim Alouini, Fellow, IEEE,
}

\begin{abstract}
Antenna selection for full-duplex communication between two nodes, each equipped with a predefined number of antennae and transmit/receive chains, is studied. Selection algorithms are proposed based on magnitude, orthogonality, and determinant criteria. The algorithms are compared to optimal selection obtained by exhaustive search as well as random selection, and are shown to yield performance fairly close to optimal at a much lower complexity. Performance comparison for a Rayleigh fading symmetric channel reveals that selecting a single transmit antenna is best at low signal-to-noise ratio (SNR), while selecting an equal number of transmit and receive antennae is best at high SNR.
\end{abstract}

Index Terms-MIMO; two-way; antenna selection; full duplex.

\section{INTRODUCTION}

$\mathbf{F}$ OLLOWING recent demonstrations in [1], [2] e.g., FullDuplex (FD) two-way communication (TWC) is now no longer considered impractical and is shown to have potentially double throughput than conventional half duplex (HD) communication [3], [4]. This has made FD a key technology for 5G systems, and has lead to significant interest into finding novel and optimal ways for better resource utilisation.

Resource management is of significant importance for enhancing FD TWC systems [5]. Antenna selection is among resource management techniques of practical interest [6], [7]. To the authors' knowledge, only few research works concentrating on FD TWC systems investigate antennae selection. For instance, [8] considers the selection of one transmit (Tx) and one receive $(\mathrm{Rx})$ antenna at each node equipped with 2 antennae (Fig. 1a). This has been generalised in [9] to the case with $N$-antenna nodes, where a nearly optimal low-complexity algorithm was proposed (Fig. 1b). Those selection algorithms are suitable for nodes equipped with single $\mathrm{Tx} / \mathrm{Rx}$ chains. What if nodes are equipped with multiple such chains?

In this letter, we consider the selection of a given number of $\mathrm{Tx}$ and $\mathrm{Rx}$ antennae at two nodes, each equipped with a given number of $T x / R x$ chains and antennae that can be used for either transmission or reception (Fig. 1c). This work thus

This publication is based upon work supported by the King Abdullah University of Science and Technology (KAUST) Office of Sponsored Research (OSR) under Award No. URF/1/2221.

D. G. Wilson-Nunn is with the Alan Turing Institute, British Library, 96 Euston Road, London, NW1 2DB. Email dwilson-nunn@turing.ac.uk.

A. Chaaban and M.-S. Alouini are with KAUST, Computer, Electrical and Mathematical Science and Engineering Division (CEMSE), Thuwal 239556900, Saudi Arabia. Email: \{anas.chaaban,slim.alouini\}@ kaust.edu.sa.

A. Sezgin is with the Chair of Digital Communication Systems, RuhrUniversität Bochum, Universitätsstraße 150, 44780 Bochum, Germany. Email: aydin.sezgin@rub.de.

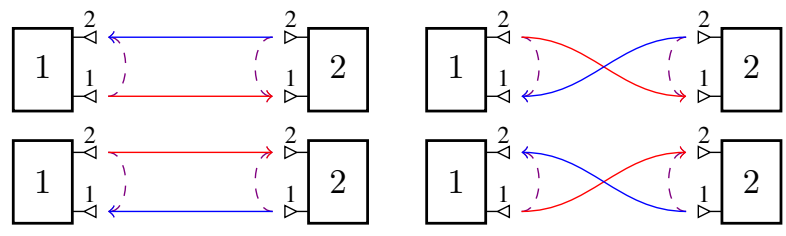

(a) The algorithm in [8] selects from these four systems.

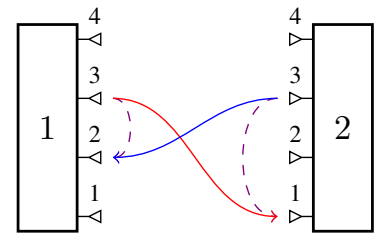

(b) Selection considered in [9].

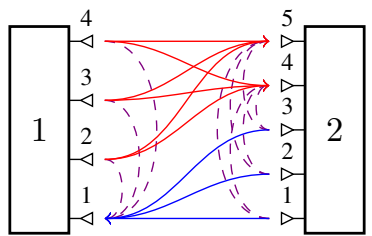

(c) Selection considered herein.
Fig. 1: Visualisation of the algorithms proposed in [8], [9] and this paper showing selected channels and self-interference.

extends [9] to systems with multiple Tx/Rx chains, wherein the problem becomes that of sub-matrix selection. The scalar selection algorithms in [9] can not be used for this case. Our main contribution is thus proposing distributed nearoptimal polynomial-complexity antenna selection algorithms, where antennae are successively chosen so as to maximise the achievable rate following magnitude, orthogonality, or determinant selection criteria. The algorithms' low complexities make them suitable for computationally-limited systems with a fixed number of Tx/Rx chains and antennae at each node.

\section{System Model and Problem Formulation}

We consider a TWC system with nodes 1 and 2 (Fig. 1c), where node $i \in\{1,2\}$ is equipped with $N_{i}$ antennae, $l_{i}$ Tx chains, and $m_{i}=N_{i}-l_{i}$ Rx chains. While these numbers are fixed, the association between $\mathrm{Tx} / \mathrm{Rx}$ chains and antennae is flexible, and can be optimised depending on the channel between the two nodes.

The channel coefficient from the $j^{\text {th }}$ antenna at node 1 to the $i^{\text {th }}$ antenna at node 2 is denoted by $h_{i j}$, and all coefficients are collected in the matrix $\mathbf{H} \in \mathbb{C}^{N_{2} \times N_{1}}$. This matrix is known at both nodes, and preserves the same value during a transmission block. We assume channel reciprocity, i.e., the channel from node 2 to 1 is given by the $\mathbf{H}^{\mathrm{H}}$. The selected channel matrix from node 1 to node 2 is denoted $\mathbf{H}_{1} \in \mathbb{C}^{m_{2} \times l_{1}}$, while that from node 2 to node 1 is denoted $\mathbf{H}_{2} \in \mathbb{C}^{m_{1} \times l_{2}}$. The transmit power of each antenna is denoted by $P$. 
The nodes simultaneously transmit and receive signals at the same frequency band, resulting in self interference (SI). We assume that multi-antenna SI cancellation is employed at each node [10]. This can be a combination of passive methods using directional antennae and/or shielding [11] or Balun transformers [12], and active methods using analog and digital circuitry [13]. The residual SI is assumed to have equal average power $\sigma_{i}^{2}$ at each antenna at node $i$, due to a symmetric design. The thermal noise power is $\sigma^{2}$, and thus, the total noise power at each receiving antenna at node $i$ is $\sigma^{2}+\sigma_{i}^{2}$. An achievable rate under these conditions is

$R\left(\mathbf{H}_{1}, \mathbf{H}_{2}\right)=\log \left|\mathbf{I}_{m_{2}}+\gamma_{1} \mathbf{H}_{1} \mathbf{H}_{1}^{\mathrm{H}}\right|+\log \left|\mathbf{I}_{m_{1}}+\gamma_{2} \mathbf{H}_{2} \mathbf{H}_{2}^{\mathrm{H}}\right|$,

where $\mathbf{I}_{l}$ denotes the $l \times l$ identity matrix and $\gamma_{i}=\frac{P}{\sigma^{2}+\sigma^{2}}$. This is obtained by invoking the worst-case noise lemma [14] leading to Gaussian noise (as in [10], [12], [13]), and by ignoring noise correlation in the decoding process.

The capacity of the resulting channel, modelled by $\mathbf{H}$, is the solution of the following discrete maximisation problem:

$$
\begin{array}{lrrrl}
\max . & R\left(\mathbf{U}_{2} \mathbf{H} \mathbf{V}_{1}, \mathbf{U}_{1} \mathbf{H}^{\mathrm{H}} \mathbf{V}_{2}\right) & \\
\text { s.t. } & \mathbf{U}_{i} \in \mathbb{F}_{2}^{m_{i} \times N_{i}}, & \mathbf{I}_{m_{i}}=\mathbf{U}_{i} \mathbf{U}_{i}^{\top}, & \mathbf{U}_{i} \mathbf{V}_{i}=\mathbf{0}_{m_{i} \times l_{i}}, \\
& \mathbf{V}_{i} \in \mathbb{F}_{2}^{N_{i} \times l_{i}}, & \mathbf{I}_{l_{i}}=\mathbf{V}_{i}^{\top} \mathbf{V}_{i}, & i=1,2 .
\end{array}
$$

Here, $\mathbf{0}_{m_{i} \times l_{i}}$ is an $m_{i} \times l_{i}$ zero matrix. The matrices denoted by $\mathbf{V}_{j}$ select the $\mathrm{Tx}$ antennae for each node, whilst the matrices denoted $\mathbf{U}_{i}$ select the $\mathrm{Rx}$ antennae.

\section{Selection Algorithms}

The discrete optimisation (1) is a combinatorial problem. Solving this exhaustively by calculating the rate for each possible $\mathbf{U}_{2}$ and $\mathbf{V}_{1}$ has prohibitive complexity. Consider the scenario $N_{2}=N_{1}=N$ and $l_{1}=m_{2}=\frac{N}{2}=\ell$ (denoted worst case scenario henceforth). There are $\left(\begin{array}{c}N \\ \ell\end{array}\right)$ different possibilities for $\mathbf{U}_{2}$ and $\mathbf{V}_{1}$. Using Sterling's formula, the complexity of exhaustive search is $O\left(2^{2 N}\right)$, which is impractical even for moderate $N$. Instead, in this section we propose suboptimal polynomial complexity selection algorithms, which we later show to provide fairly good performance. We start with some analysis which motivates the algorithms.

Consider a channel matrix $\mathbf{H} \in \mathbb{C}^{4 \times 4}$ where we wish to pick $\mathbf{H}_{1} \in \mathbb{C}^{2 \times 2}$, i.e., $l_{1}=m_{2}=2$. Notice that both $\mathbf{I}_{2}$ and $\mathbf{H}_{1} \mathbf{H}_{1}^{\mathrm{H}}$ are positive semi-definite. Then, by Minkowski's determinant theorem, we know that

$$
\left|\mathbf{I}_{2}+\gamma_{1} \mathbf{H}_{1} \mathbf{H}_{1}^{\mathrm{H}}\right| \geq\left|\mathbf{I}_{2}\right|+\left|\gamma_{1} \mathbf{H}_{1} \mathbf{H}_{1}^{\mathrm{H}}\right|=1+\left|\gamma_{1} \mathbf{H}_{1} \mathbf{H}_{1}^{\mathrm{H}}\right| .
$$

So, increasing $\left|\mathbf{H}_{1} \mathbf{H}_{1}^{\mathrm{H}}\right|$ increases the lower bound above. Now assume that the vector of channels $\mathbf{h}_{1}=\left(h_{11}, h_{12}\right)$ is initially selected. Then, we wish to pick $\mathbf{a}=\left(a_{1}, a_{2}\right)$ to form $\mathbf{H}_{1}=$ $\left[\begin{array}{c}\mathbf{h}_{1} \\ \mathbf{a}\end{array}\right]$, where $\mathbf{a} \in\left\{\mathbf{h}_{2}, \mathbf{h}_{3}, \mathbf{h}_{4}\right\}$ and $\mathbf{h}_{i}=\left(h_{i 1}, h_{i 2}\right)$. We have

$$
\left|\mathbf{H}_{1} \mathbf{H}_{1}^{\mathrm{H}}\right|=\left\|\mathbf{h}_{1}\right\|^{2}\|\mathbf{a}\|^{2}-\left|\mathbf{h}_{1} \mathbf{a}^{\mathrm{H}}\right|^{2} .
$$

Now one can choose a based on one of the following criteria:

1) Magnitude: We pick $\mathbf{a}=\arg \max _{\mathbf{h}_{i}}\left\|\mathbf{h}_{i}\right\|$. This maximises the first term on the right-hand side (RHS) of (3).

2) Orthogonality: We pick a which is closest to orthogonal to $\mathbf{h}_{1}$, i.e., $\mathbf{a}=\arg \min _{\mathbf{h}_{i}}\left|\mathbf{h}_{1} \mathbf{h}_{i}^{\mathrm{H}}\right|$, minimising the

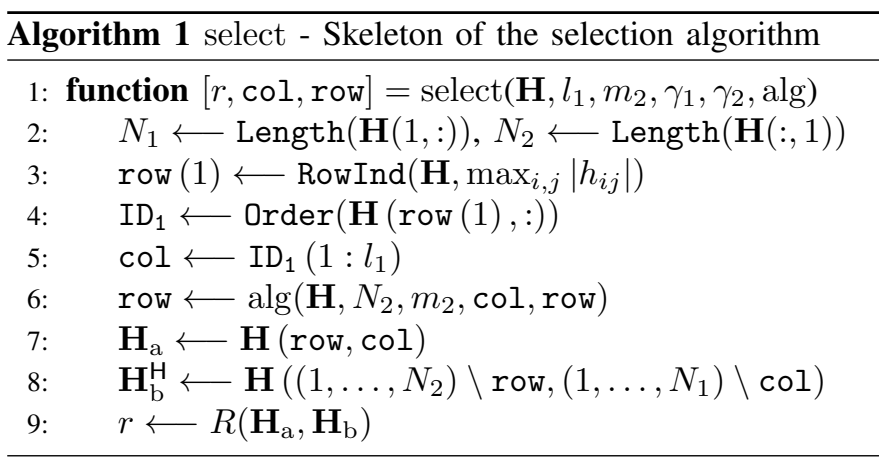

second term on the RHS of (3). Alternatively, we can pick $\mathbf{a}=\arg \max _{\mathbf{h}_{i}}\left|\mathbf{g}_{1} \mathbf{h}_{i}^{\mathrm{H}}\right|$ where $\mathbf{g}_{1} \in \mathbb{C}^{1 \times 2}$ satisfies $\mathbf{h}_{1} \mathbf{g}_{1}^{\mathrm{H}}=0$. The advantage is that this variant selects $\mathbf{a}$ which is potentially near orthogonal to $\mathbf{h}_{1}$ and of large magnitude. We stick to this variant henceforth.

3) Determinant: We pick $\mathbf{a}=\arg \max _{\mathbf{h}_{i}}\left|\left[\begin{array}{c}\mathbf{h}_{1} \\ \mathbf{a}\end{array}\right]\left[\begin{array}{c}\mathbf{h}_{1} \\ \mathbf{a}\end{array}\right]^{\mathrm{H}}\right|$, which maximises the left-hand side of (3).

This intuition can be extended to matrices of higher dimensions. Based on this insight, we propose to construct $\mathbf{H}_{1}$ by increasing its size gradually row by row, by selecting among vectors which share the same columns according to the above criteria. This can be also applied column-wise. This stepby-step procedure leads to polynomial complexity which is desirable. The same idea can be applied either for $\mathbf{H}_{1}$ or $\mathbf{H}_{2}$. Although this discussion only takes one submatrix into consideration, numerical results show that this leads to nearoptimal results as we shall see. Furthermore, in a symmetric scenario $\left(N_{1}=N_{2}, \gamma_{1}=\gamma_{2}, l_{1}=l_{2}\right)$, we can transmit over channel $\mathbf{H}_{1}\left(\mathbf{H}_{2}\right)$ from node 1 to 2 (2 to 1$)$ half the time, and over channel $\mathbf{H}_{2}^{\mathrm{H}}\left(\mathbf{H}_{1}^{\mathrm{H}}\right)$ from node 1 to 2 (2 to 1 ) half the time, leading to symmetric performance while preserving the sum rate $R\left(\mathbf{H}_{1}, \mathbf{H}_{2}\right)$. We introduce the algorithms next.

Notation: In what follows, we use $\operatorname{Order}(\mathbf{v})$ to denote the indexes of the elements of the vector $\mathbf{v}$ sorted in decreasing order, Length $(\mathbf{v})$ to denote its number of elements, $\mathbf{v}(\mathbf{i})$ to denotes the vector formed by the elements of $\mathbf{v}$ indexed by $\mathbf{i}$, and $\mathbf{v}_{1} \backslash \mathbf{v}_{2}$ denotes the elements of $\mathbf{v}_{1}$ not in $\mathbf{v}_{2}$. The notation $n: m$ denotes the vector $(n, n+1, \ldots, m)$. For a matrix $\mathbf{A}$, RowInd $(\mathbf{A}, k)$ denotes the index of the row containing the element $k, \mathbf{B}=\operatorname{NullSp}(\mathbf{A})$ is the null space of $\mathbf{A}$, i.e., $\mathbf{A B}=0$, and $\mathbf{A}(\mathbf{i}, \mathbf{j})$ is the submatrix of $\mathbf{A}$ formed by the rows and columns indexed in $\mathbf{i}$ and $\mathbf{j}$, respectively, where if $\mathbf{i}$ (j) is given by ":", then all the rows (columns) of $\mathbf{A}$ are used.

\section{A. Selection Algorithm Based Upon Magnitude}

Since the algorithms we describe next share the same skeleton, we start by introducing this skeleton in Alg. 1. In this algorithm, we wish to select $l_{1}$ Tx antennae at node 1 and $m_{2} \mathrm{Rx}$ antennae at node 2 . We start similar to [9] by selecting the largest element of $\mathbf{H}$ (step 3). Then, we select the next $l_{1}-1$ largest elements in the same row, thus selecting $l_{1} \mathrm{Tx}$ antennae at node 1 (step 5). This fixes $\mathbf{V}_{1}$ in (1). To finalise the selection, we need to fix the $\mathrm{Rx}$ antennae at node 2 , i.e., $\mathbf{U}_{2}$. This is done in step 6 , which depends on the input alg $\in\{$ magSearch, nullSearch, detSearch $\}$, corresponding to 

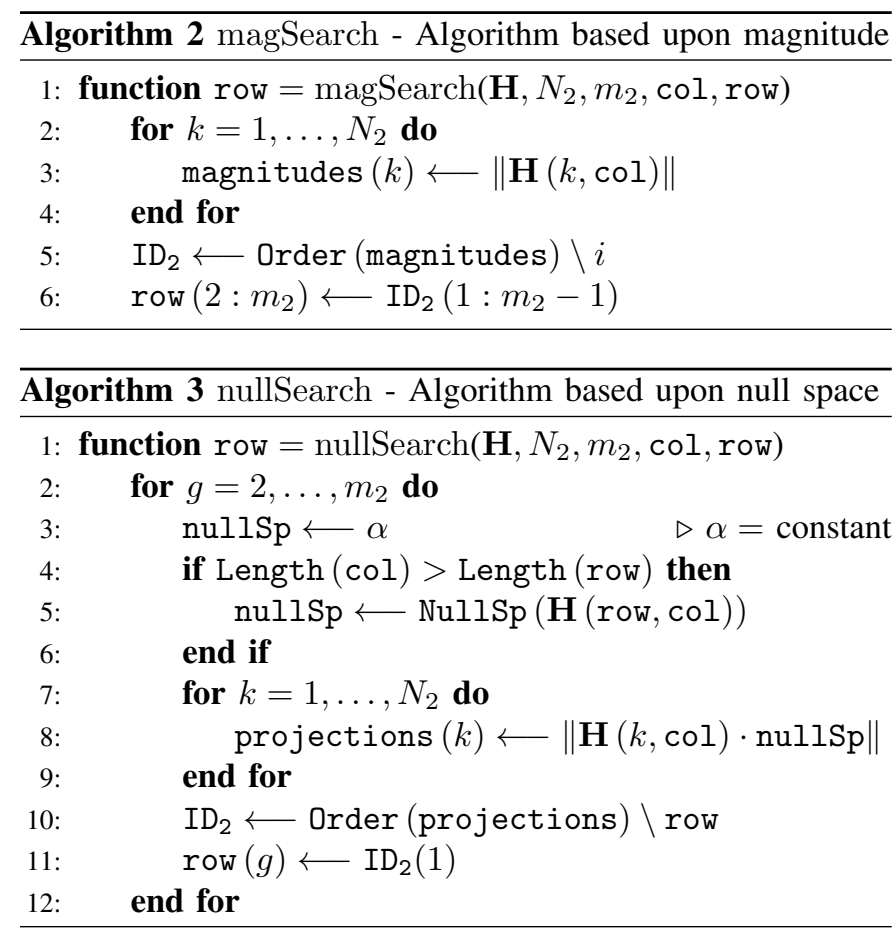

the 3 selection criteria above, respectively. Depending on alg, a number of rows will be selected, consequently fixing $\mathbf{H}_{1}$ and $\mathbf{H}_{2}$ by steps 7 and 8 . Finally, the algorithm returns the achievable rate and the selected antennae.

The simple initialization in Alg. 1 (step 3) is chosen as a low-complexity compromise, instead of starting with the row which has the largest vector of length $l_{1}$. Namely, for the worst case scenario, this initialization has complexity $O\left(N^{2} \log (N)\right)$ whereas the latter alternative has exponential complexity. Thus, the overall complexity of Alg. 1 is $O\left(N^{2} \log (N)\right)$ plus the complexity of step 6 .

Note that Alg. 1 starts by selecting Tx antennae at node 1 (steps 3-5). One could instead start by selecting Tx antennae at node 2 , Rx antennae at node 1 , or Rx antennae at node 2 . The best solution is the best of the following 4 possibilities:

$$
\begin{array}{ll}
\left(\mathbf{H}, l_{1}, m_{2}, \gamma_{1}, \gamma_{2}, \text { alg }\right), & \left(\mathbf{H}, m_{1}, l_{2}, \gamma_{2}, \gamma_{1}, \text { alg }\right), \\
\left(\mathbf{H}^{\mathrm{H}}, m_{2}, l_{1}, \gamma_{1}, \gamma_{2}, \text { alg }\right), & \left(\mathbf{H}^{\mathrm{H}}, l_{2}, m_{1}, \gamma_{2}, \gamma_{1}, \text { alg }\right) .
\end{array}
$$

This only slightly affect complexity since it runs Alg. 1 four times independent of $N_{1}$ and $N_{2}$.

The magSearch algorithm is described in Alg. 2. Here, given the selected row in Alg. 1, we select an $m_{2} \times l_{1}$ submatrix by calculating the magnitudes of the remaining rows of $\mathbf{H V}_{1}$ and selecting the $m_{2}-1$ rows with the largest magnitudes. The complexity of Alg. 2 for the worst case scenario is $O\left(N^{2}\right)$ owing to step 3 which has complexity $O(N)$ and is carried out $N$ times. Thus, the overall complexity of Alg. 1 combined with Alg. 2 remains $O\left(N^{2} \log (N)\right)$.

\section{B. Selection Algorithm Based Upon Null Space}

Selection based on the null space criterion is achieved by setting alg $=$ nullSearch in Alg. 1. As described in Alg. 3, nullSearch selects the best $m_{2} \times l_{1}$ submatrix by iteratively

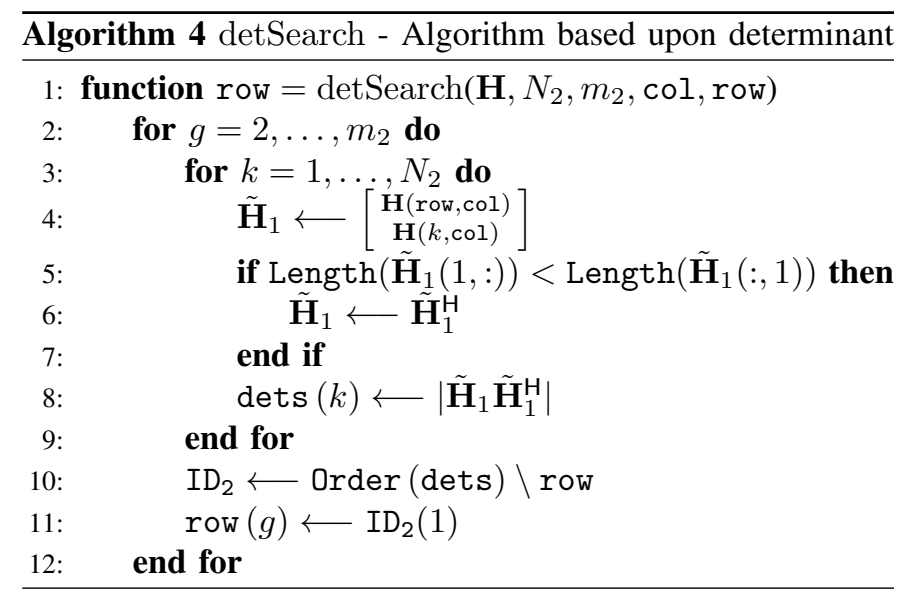

finding rows of $\mathbf{H V}_{1}$ with the greatest projection onto the null space of the matrix formed by the previously selected rows of $\mathbf{H V}_{1}$. Since the null space of $\mathbf{H}$ (row, col) exists (almost surely) only if it has more columns than rows, Alg. 3 sets the variable nullSp to a constant scalar otherwise (step 3). In this case, step 8 computes the magnitude of the subrows, and the one with the largest magnitude is selected. The largest rate possible using this selection method is computed by running Alg. 1 with inputs (4) and alg replaced with nullSearch, and then selecting the best outcome. The overall complexity of Alg. 1 combined with Alg. 3 for the worst case scenario is $O\left(N^{4}\right)$, owing to steps 5 and 8 in Alg. 3 which have complexities $O\left(N^{3}\right)$ and $O\left(N^{2}\right)$, and are executed up to $N$ and $N^{2}$ times, respectively.

\section{Selection Algorithm Based Upon Determinant}

This selection is performed by setting alg $=$ detSearch in Alg. 1, where detSearch is described in Alg. 4. The algorithm fixes $\mathbf{U}_{2}$ by iteratively finding rows of $\mathbf{H} \mathbf{V}_{1}$ which maximise $\left|\tilde{\mathbf{H}}_{1} \tilde{\mathbf{H}}_{1}^{\mathrm{H}}\right|$ where $\tilde{\mathbf{H}}_{1}$ is formed by appending a new row to the previously selected rows of $\mathbf{H V}_{1}$ (step 4). The algorithm transposes $\tilde{\mathbf{H}}_{1}$ if it has more rows than columns (steps 5-7) to guarantee a nonzero $\left|\tilde{\mathbf{H}}_{1} \tilde{\mathbf{H}}_{1}^{\mathrm{H}}\right|$. This is permissible because $\left|\mathbf{I}_{m_{2}}+\gamma_{1} \mathbf{H}_{1} \mathbf{H}_{1}^{\mathrm{H}}\right|=\left|\mathbf{I}_{l_{1}}+\gamma_{1} \mathbf{H}_{1}^{\mathrm{H}} \mathbf{H}_{1}\right|$ by Sylvester's determinant identity. The largest rate is obtained by running Alg. 1 using the inputs in (4) with alg replaced with detSearch. The overall complexity of Alg. 1 combined with Alg. 4 for the worst case is $O\left(N^{5}\right)$, owing to step 8 in Alg. 4 which has complexity $O\left(N^{3}\right)$ and is carried out at most $N^{2}$ times.

\section{Simulation Results}

To determine the effectiveness of the proposed algorithms, some simulations are in order. Herein, we consider a block fading scenario, where $h_{i j}$ is complex Gaussian with zero mean and unit variance (Rayleigh fading). Furthermore, as in [9], we write the residual SI power as $\sigma_{i}^{2}=\eta P_{i}$ for some $\eta \in[0,1]$, where in our case $P_{i}=l_{i} P$ is the total Tx power of node $i$, since each antenna receives SI from all Tx antennae.

Fig. 2a shows the average rate $\mathbb{E}\left[R\left(\mathbf{H}_{1}, \mathbf{H}_{2}\right)\right]$ obtained by the proposed algorithms, along with exhaustive search and random selection when $N_{1}=N_{2}=N, l_{1}=m_{2}=2$ and 


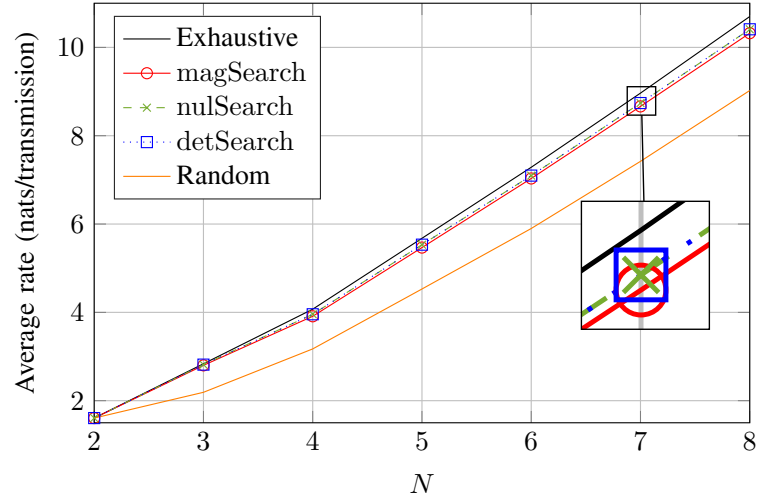

(a) Proposed algorithms, exhaustive search, and random selection: $N_{1}=N_{2}=N, l_{1}=m_{2}=2$ and $l_{2}=m_{1}=N-2$.

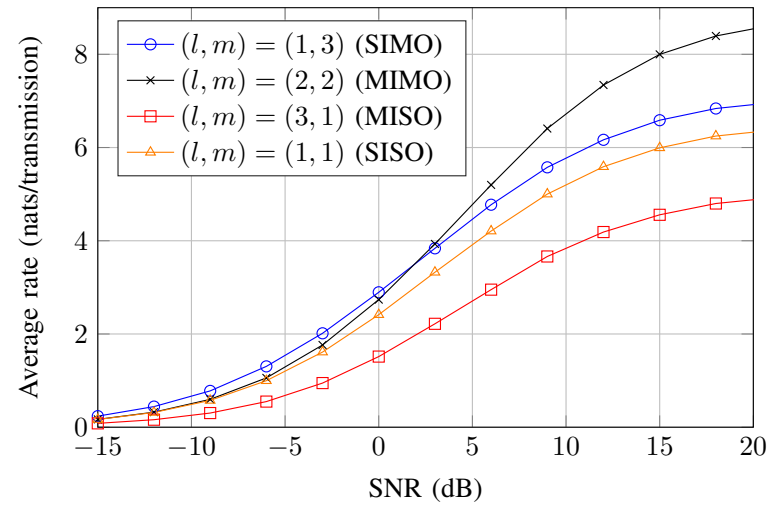

(b) magSearch compared with "serial-max" [9]: $N_{1}=N_{2}=4$.

Fig. 2: Average rates for a Rayleigh fading channel.

$l_{2}=m_{1}=N-2$, for $N=2, \ldots, 8(N=2$ corresponds to an HD system). We set $P=\sigma^{2}=1$ and $\eta=0.1$. Interestingly, the detSearch and nullSearch algorithms achieve similar performance, achieving $>97 \%$ of the optimal rate, significantly better than random selection. On the other hand, the magSearch algorithm achieves performance fairly close to optimal, at a much lower complexity (see Table I). This demonstrates the effectiveness of the proposed algorithms.

Fig. $2 b$ shows average rates versus SNR for a channel with $N_{1}=N_{2}=4, \eta=0.1$, and $\sigma^{2}=1$. The number of selected antennae satisfies $l_{1}=l_{2}=l, m_{1}=m_{2}=m$, with: $(l, m)=(1,3)$ (SIMO), $(l, m)=(2,2)($ MIMO), $(l, m)=(3,1)$ (MISO), and $(l, m)=(1,1)$ (SISO). We use the "serial-max" selection approach [9] in the last case, while we restrict our attention to the magSearch algorithm in the first 3 cases due to its good performance (Fig. 2a) and low complexity. For a fair comparison, we consider equal total Tx power $P_{t}$ for all systems, i.e., the power per antenna is $\frac{P_{t}}{l_{i}}$. The SNR is defined as $P_{t} / \sigma^{2}$. Due to the system definition, all 4 cases have the same noise variance $\sigma^{2}+\sigma_{i}^{2}=1+\eta P$. The SIMO and SISO cases have the same Tx power per antenna $P_{t}$, but SIMO is superior due to its array gain. SIMO is also better than MIMO at low SNR due to the power gain; MIMO has Tx power per antenna $P_{t} / 2$. However, MIMO soon outperforms SIMO due to its higher multiplexing gain. The MISO case is inferior because it spreads $P_{t}$ over 3 antennae leading to lower Rx SNR. This suggests using a single Tx antenna at low SNR,
TABLE I: Comparison of the algorithms' complexities.

\begin{tabular}{c||c|c|c|c}
\hline Alg. & Exh. & detS. & nullS. & magS. \\
\hline Complexity & $O\left(2^{2 N}\right)$ & $O\left(N^{5}\right)$ & $O\left(N^{4}\right)$ & $O\left(N^{2} \log (N)\right)$ \\
\hline
\end{tabular}

versus an equal number of $\mathrm{Tx}$ and $\mathrm{Rx}$ antennae at high SNR.

\section{CONCLUSION}

We have proposed polynomial-complexity two-way link selection algorithms for FD MIMO scenarios that attempt to maximise the information transmission rate of the system. The algorithms provide near optimal performance at a reasonable complexity. This makes those algorithms suitable for implementation in systems with computational limitations. As an extension, it would be interesting to study a similar problem under a total power constraint instead of a per-antenna power constraint. The proposed algorithms can be used in this case, but better solutions can be obtained by considering antenna selection and power allocation jointly.

\section{REFERENCES}

[1] J. I. Choi, M. Jain, K. Srinivasan, P. Levis, and S. Katti, "Achieving single channel, full duplex wireless communication," in Proc. 16th Ann. Int. Conf. Mobile Comput. Netw., Chicago, Il, USA, Sep. 2010, pp. 1-12.

[2] D. Bharadia, E. McMilin, and S. Katti, "Full duplex radios," in Proc. ACM SIGCOMM, Hong Kong, China, Aug. 2013, pp. 375-386.

[3] T. Riihonen, S. Werner, and R. Wichman, "Hybrid full-duplex/halfduplex relaying with transmit power adaptation," IEEE Trans. Wireless Commun., vol. 10, no. 9, pp. 3074-3085, Sep. 2011.

[4] A. Chaaban and A. Sezgin, "Multi-way Communications: An Information Theoretic Perspective," Found. Trends Commun. Inf. Theory, vol. 12, no. 3-4, pp. 185-371, 2015.

[5] L. Song, Y. Li, and Z. Han, "Resource allocation in full-duplex communications for future wireless networks," IEEE Wireless Commun. Mag., vol. 22, no. 4, pp. 88-96, Aug. 2015.

[6] M. Gharavi-Alkhansari and A. B. Gershman, "Fast antenna subset selection in MIMO systems," IEEE Trans. Signal Process., vol. 52, no. 2, pp. 339-347, Feb. 2004.

[7] J. G. Klotz and A. Sezgin, "Antenna selection criteria for interference alignment," in Proc. 21st Int. Symp. Pers. Indoor and Mobile Radio Commun., Sep. 2010, pp. 527-531.

[8] M. Zhou, H. Cui, L. Song, and B. Jiao, "Transmit-receive antenna pair selection in full duplex systems," IEEE Wireless Commun. Lett., vol. 3, no. 1, pp. 34-37, Feb. 2014.

[9] M. Zhou, L. Song, Y. Li, and X. Li, "Simultaneous bidirectional link selection in full duplex MIMO systems," IEEE Trans. Wireless Commun., vol. 14, no. 7, pp. 4052-4062, Jul. 2015.

[10] M. Duarte, A. Sabharwal, V. Aggarwal, R. Jana, K. K. Ramakrishnan, C. W. Rice, and N. K. Shankaranarayanan, "Design and characterization of a full-duplex multiantenna system for WiFi networks," IEEE Trans. Veh. Technol., vol. 63, no. 3, pp. 1160-1177, Mar. 2014.

[11] E. Everett, A. Sahai, and A. Sabharwal, "Passive self-interference suppression for full-duplex infrastructure nodes," IEEE Trans. Wireless Commun., vol. 13, no. 2, pp. 680-694, Feb. 2014.

[12] M. Jain, J. I. Choi, T. Kim, D. Bharadia, S. Seth, K. Srinivasan, P. Levis, S. Katti, and P. Sinha, "Practical, real-time, full duplex wireless," in Proc. 17th Ann. Int. Conf. Mobile Comput. Netw., Las Vegas, NV, USA, Sep. 2011, pp. 301-312.

[13] M. Duarte, C. Dick, and A. Sabharwal, "Experiment-driven characterization of full-duplex wireless systems," IEEE Trans. Wireless Commun., vol. 11, no. 12, pp. 4296-4307, Dec. 2012.

[14] S. Diggavi and T. Cover, "The worst additive noise under a covariance constraint," IEEE Tran. Inf. Theory, vol. 47, no. 7, pp. 3072-3081, Nov. 2001. 